\title{
The impact of personalised therapies on respiratory medicine
}

\author{
J. Stuart Elborn
}

ABSTRACT: Stratified approaches to treating disease are very attractive, as efficacy is maximised by identifying responders using a companion diagnostic or by careful phenotyping. This approach will spare non-responders form potential side-effects. This has been pioneered in oncology where single genes or gene signatures indicate tumours that will respond to specific chemotherapies. Stratified approaches to the treatment of asthma with biological therapies are currently being extensively studied. In cystic fibrosis (CF), therapies have been developed that are targeted at specific functional classes of mutations. Ivacaftor, the first of such therapies, potentiates dysfunctional cystic fibrosis transmembrane conductance regulator (CFTR) protein Class III mutations and is now available in the USA and some European countries. Pivotal studies in patients with a G551D mutation, the most common Class III mutation, have demonstrated significant improvements in clinically important outcomes such as spirometry and exacerbations. Sweat chloride was significantly reduced demonstrating a functional effect on the dysfunctional CFTR protein produced by the G551D mutation. Symptom scores are also greatly improved to a level that indicates that this is a transformational treatment for many patients. This stratified approach to the development of therapies based on the functional class of the mutations in CF is likely to lead to new drugs or combinations that will correct the basic defect in many patients with CF.

\section{KEYWORDS: Cystic fibrosis, personalised medicine, potentiator therapy}

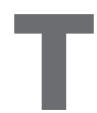
reatment approaches for respiratory diseases have generally involved all patients with a given condition receiving the same therapy. This assumes that the underlying disease pathophysiology is similar for all patients and that all patients will have the same treatment response to a therapy. As this is invariably not the case, there are increased efforts to develop personalised therapies. The series of articles in the current issue of the European Respiratory Review has explored this approach in some detail, describing its application to respiratory diseases, in general, and cystic fibrosis, in particular [1-4].

Early examples of personalised or stratified medicine mostly stem from the field of oncology [2]. An archetypal example is trastuzumab, better known as Herceptin $®$, for adjunctive treatment of patients with human epidermal growth factor receptor 2 (HER2) gene-amplified breast cancer [5]. Two clinical trials have shown that trastuzumab was associated with a $33 \%$ reduction in the risk of death $(p=0.015)$ [6] and improved 3-yr recurrence-free survival [7], compared to patients who did not receive therapy. In the field of endocrinology, PEARSON et al. [8] reported that diabetic patients with mutations in the gene encoding a subunit of the pancreatic ATP-sensitive channel responded better to sulfonylurea therapy treatment than insulin.

Examples of personalised medicine in the field of respiratory medicine are also mostly in the area of oncology, where knowledge of tumour-related mutations has been used to refine the selection of chemotherapy. The kinase inhibitor crizotinib was specifically developed to treat nonsmall cell lung cancer (NSCLC) patients who have a chromosomal transformation that activates the anaplastic lymphoma kinase $(A L K)$ gene [9]. Updated results of a phase I trial assessing crizotinib in ALK-positive NSCLC patients showed that $87(60.8 \%)$ out of 143 patients had an objective response (95\% CI 52.3-68.9\%) and median progression-free survival was 9.7 months (95\% CI 7.7-12.8 months) [10].

Extending these approaches to non-malignant respiratory disease presents exciting possibilities, and has already led to new approaches to phenotyping in areas such as chronic obstructive

\section{AFFILIATION}

Centre for Infection and Immunity, School of Medicine, Dentistry and Biomedical Sciences, Queens

University, Belfast, UK.

\section{CORRESPONDENCE}

J.S. Elborn

Centre for Infection and Immunity, School of Medicine, Dentistry and Biomedical Sciences

Queens University

Health Sciences Building

97 Lisburn Road

Belfast

BT9 7BL

UK

E-mail: s.elborn@qub.ac.uk

Received:

Dec 202012

Accepted after revision:

Jan 152013

PROVENANCE

Publication of this peer-reviewed article was supported by Vertex

Pharmaceuticals Inc., USA (principal sponsor, European Respiratory Review issue 127). 
TABLE 1 Mechanism of action and targets of emerging cystic fibrosis transmembrane conductance regulator (CFTR) modulators

\begin{tabular}{|c|c|c|c|c|c|}
\hline $\begin{array}{l}\text { Mutation } \\
\text { class }\end{array}$ & Nature of defect & Example mutations & Pancreatic function ${ }^{\#}$ & Modulator strategy & Agents in development \\
\hline II & Trafficking & F508del & Insufficient & $\begin{array}{c}\text { Corrector } \\
\text { Corrector plus potentiator }\end{array}$ & $\begin{array}{l}\text { VX-809 plus ivacaftor }{ }^{+} \\
\text {VX-661 plus ivacaftor }{ }^{+}\end{array}$ \\
\hline IV & Channel conductance & $\mathrm{R} 117 \mathrm{H}$ & Sufficient & Potentiator?" & No clinical data available \\
\hline v & Low transcript levels & $3849+10 \mathrm{~kb} \mathrm{C}->\mathrm{T}$ & Sufficient & Potentiator?" & No clinical data available \\
\hline
\end{tabular}

FDA: US Food and Drug Administration. ${ }^{\#}$ : when in trans with a non-functional CFTR mutation; ${ }^{\circ}$ : theoretical approach based on nature of defect and known drug mechanism; ${ }^{+}$: currently in phase II trials. Reproduced from [15] with permission from the publisher.

pulmonary disease, asthma, lung fibrosis or cancer using, for example, serum markers, blood count, clinical phenotypes, somatic genotype, germinal genotype and co-morbidities. Not all of these approaches will necessarily end up being applicable in practice, but they are worthy of investigation. Multiple genome-wide association studies (GWAS) have been published in respiratory medicine, mostly relating to genes associated with asthma development or susceptibility to chronic obstructive pulmonary disease [11]. Recently, there has been increased interest in the role of interleukin (IL)-33 in asthma. IL-33 is associated with promoting a type- 1 T-helper cell response and may play a role in chronic inflammation and airway remodelling [12]. Moreover, IL-33 gene polymorphisms have been identified in various asthma GWAS analyses [2, 13, 14].

The recent identification of the ability of ivacaftor, a cystic fibrosis transmembrane conductance regulator (CFTR) modulator, to treat some patients with cystic fibrosis (CF) is the first example in which a stratified medicine approach has resulted in a major change in therapy for patients with this disease who carry the gating channel mutation, G551D [15].

\section{CFTR MODULATORS: A POTENTIAL PARADIGM FOR "PERSONALISED RESPIRATORY MEDICINE"}

Ivacaftor, formerly known as VX-770, was identified from a library of 228,000 diverse drugs using high-throughput screening [13]. The candidate agent was subsequently shown to increase the channel opening probability of G551D CFTR by approximately six-fold and to partially restore fluid regulation and cilia beating in cultured CF human bronchial epithelia with the G551D/F508del genotype [3, 16]. These results provided convincing evidence and rapidly advanced into clinical trials.

The efficacy and safety of ivacaftor was evaluated in two 48week, randomised, double-blind, placebo-controlled trials in patients aged 6-11 yrs (ENVISION study) and patients aged $\geqslant 12$ yrs (STRIVE study) who have at least one copy of the G551D-CFTR mutation [4].

Ivacaftor was shown to result in a rapid and sustained improvement in lung function through to week 24 and this effect continued through to week 48 . Sweat chloride concentration significantly decreased in both trials to levels below the diagnostic cut-off point for CF. Moreover, treated patients in the ENVISION and STRIVE studies demonstrated significant improvements in body weight. The most common reported adverse events were abdominal pain, diarrhoea, dizziness, rash, upper respiratory tract reactions, headache and bacteria in sputum.

A separate phase II trial, DISCOVER, assessed the efficacy of ivacaftor in patients homozygous for the F508del-CFTR mutation [17]. No observed clinical effect was measured between the treatment and placebo arms, suggesting that CFTR potentiation with ivacaftor alone will not benefit $C F$ patients who are homozygous for F508del-CFTR. The reason for this is clear; ivacaftor is a CFTR potentiator that targets gating defects in G551D-CFTR channels. F508del mutation defects, however, can only be repaired through use of a CFTR corrector, such as VX-808 [3].

\section{Emerging CFTR modulators}

Ivacaftor, the first CFTR modulator to be approved for clinical use, can be only prescribed to approximately $4-5 \%$ of CF patients who have the G551D mutation. If successful, other emerging CFTR therapies will hold greater promise to treat many more CF patients (table 1 ).

The read through agent, ataluren, is undergoing clinical trials to treat $\mathrm{CF}$ in patients with mutations caused by premature stop codons. Ataluren was linked to improvement in cough over 3 months and increases in CFTR activity, as measured by the nasal potential difference [18, 19]. Results of a phase III pivotal trial are expected soon and are eagerly awaited.

VX-809 (or lumicaftor) is a CFTR corrector that has been assessed in a phase IIa study of patients homozygous for the F508del-CFTR mutation. The drug, which was well tolerated with no significant safety signals, caused a moderate reduction in sweat chloride compared to placebo [20]. Surprisingly, no other clinically significant improvements were measured. Nevertheless, the trial provided a basis for investigation into the safety and efficacy of lumicaftor plus ivacaftor combination therapy in CF patients homozygous or heterozygous for the F508del-CFTR mutations [21].

\section{Impact of personalised medicine on CF care}

Based on experience with ivacaftor, the potential for CFTR modulators (alone or combined) to transform the lives of a significant number of CF patients is exciting. This optimism must 
also be guarded: many trials of promising drug candidates are still in their infancy and the long-term effect of these therapies is still unknown. Nevertheless, as personalised therapies, CFTR modulators truly represent a milestone in CF treatment.

\section{CONCLUSION}

The theoretical benefit of personalised respiratory medicine is almost beyond reproof. However, despite an increasing number of success stories, such as crizotinib, trastuzumab, imatinib and ivacaftor, the development of personalised therapies remains a significant challenge; one that is perhaps above and beyond those typically faced by the pharmaceutical industry, which also has to consider how to recoup the not-insignificant development costs in a specialised and limited market. Overcoming these challenges will involve input and consensus from funders, scientists, providers, patients and regulatory authorities. Nevertheless, personalised medicine has the genuine potential to revolutionise healthcare as we know it.

\section{STATEMENT OF INTEREST}

J.S. Elborn has received fees for consultancy and speaking from Vertex. He received payment as a grant for three clinical trials $(€ 50,000)$.

\section{REFERENCES}

1 Elborn JS. Personalised medicine for cystic fibrosis: treating the basic defect. Eur Respir Rev 2013; 22: 3-5.

2 Hall IP. Stratified medicine: drugs meet genetics. Eur Respir Rev 2013; 22: 53-57.

3 Derichs N. Targeting a genetic defect: cystic fibrosis transmembrane conductance regulator modulators modulators in cystic fibrosis. Eur Respir Rev 2013; 22: 58-65.

4 Sermet-Gaudelus I. Ivacaftor treatment in patients with cystic fibrosis and the G551D-CFTR mutation. Eur Respir Rev 2013; 22: 66-71.

5 Slamon DJ, Leyland-Jones B, Shak S, et al. Use of chemotherapy plus a monoclonal antibody against HER2 for metastatic breast cancer that overexpresses HER2. N Engl J Med 2001; 344: 783-792.

6 Romond EH, Perez EA, Bryant J, et al. Trastuzumab plus adjuvant chemotherapy for operable HER2-positive breast cancer. $N$ Engl J Med 2005; 353: 1673-1684.

7 Joensuu H, Kellokumpu-Lehtinen PL, Bono P, et al. Adjuvant docetaxel or vinorelbine with or without trastuzumab for breast cancer. N Engl J Med 2006; 354: 809-820.
8 Pearson ER, Flechtner I, Njolstad PR, et al. Switching from insulin to oral sulfonylureas in patients with diabetes due to Kir6.2 mutations. N Engl J Med 2006; 355: 467-477.

$9 \mathrm{Ou} \mathrm{SH}$, Bartlett $\mathrm{CH}$, Mino-Kenudson $\mathrm{M}$, et al. Crizotinib for the treatment of ALK-rearranged non-small cell lung cancer: a success story to usher in the second decade of molecular targeted therapy in oncology. Oncologist 2012; 17: 1351-1375.

10 Camidge DR, Bang YJ, Kwak EL, et al. Activity and safety of crizotinib in patients with ALK-positive non-small-cell lung cancer: updated results from a phase 1 study. Lancet Oncol 2012; 13: 1011-1019.

11 Todd JL, Goldstein DB, Ge D, et al. The state of genome-wide association studies in pulmonary disease: a new perspective. Am J Respir Crit Care Med 2011; 184: 873-880.

12 Prefontaine D, Nadigel J, Chouiali F, et al. Increased IL-33 expression by epithelial cells in bronchial asthma. J Allergy Clin Immunol 2010; 125: 752-754.

13 Smith DE. IL-33: a tissue derived cytokine pathway involved in allergic inflammation and asthma. Clin Exp Allergy 2010; 40: 200-208.

14 Ober C, Yao TC. The genetics of asthma and allergic disease: a 21st century perspective. Immunol Rev 2011; 242: 10-30.

15 Clancy JP, Jain M. Personalized medicine in cystic fibrosis: dawning of a new era. Am J Respir Crit Care Med 2012; 186: 593-597.

16 Van Goor F, Hadida S, Grootenhuis PD, et al. Correction of the F508del-CFTR protein processing defect in vitro by the investigational drug VX-809. Proc Nat Acad Sci USA 2011; 108: 18843-18848.

17 Flume PA, Liou TG, Borowitz DS, et al. Ivacaftor in subjects with cystic fibrosis who are homozygous for the F508del-CFTR mutation. Chest 2012; 142: 718-724.

18 Kerem E, Hirawat S, Armoni S, et al. Effectiveness of PTC124 treatment of cystic fibrosis caused by nonsense mutations: a prospective phase II trial. Lancet 2008; 372: 719-727.

19 Sermet-Gaudelus I, Boeck KD, Casimir GJ, et al. Ataluren (PTC124) induces cystic fibrosis transmembrane conductance regulator protein expression and activity in children with nonsense mutation cystic fibrosis. Am J Respir Crit Care Med 2010; 182: $1262-1272$.

20 Clancy JP, Rowe SM, Accurso FJ, et al. Results of a phase IIa study of VX-809, an investigational CFTR corrector compound, in subjects with cystic fibrosis homozygous for the F508del-CFTR mutation. Thorax 2012; 67: 12-18.

21 Pettit RS. Cystic fibrosis transmembrane conductance regulatormodifying medications: the future of cystic fibrosis treatment. Ann Pharmacother 2012; 46: 1065-1075. 